\title{
Vehicle Platooning System with Tensor-based Space-Time Coding Schemes
}

\author{
Bárbara da Silva Oliveira, Michel Gonzaga dos Santos e Walter da Cruz Freitas Júnior
}

\begin{abstract}
In this article, we discuss the platooning system and propose models for its implementation based on multilinear algebra. It is investigated a model with Khatri-Rao Space-Time (KRST) coding and another model that combine a Tensor SpaceTime (TST) and Kronecker Space-Time (KronST) codings, using Parallel Factor Analysis (PARAFAC) and Tucker decompositions. A performance analysis is carried out to illustrate and compare the efficiency of the presented algorithms.
\end{abstract}

Keywords-Platooning, MIMO, TST, KRST, KronST.

\section{INTRODUCTION}

Platooning is a cooperative driving system, where semiautonomous or autonomous vehicles are orderly conducted, allowing a more secure, sustainable and optimized traffic [1]. This system emerge from a multidisciplinary field, which implies that several sub-problems can be considered. An important one consists of proposing solutions to ensure the reliability and efficiency of the communication between the vehicles [1]. The use of Multiple-Input Multiple-Output (MIMO), i.e. multiple transmit and receive antennas, in mobile communications has been widely exploited since it provides spatial multiplexing and improves space diversity, which makes it a good candidate solution for this task.

In this paper, we consider a Platooning system composed by $K$ vehicles. The information flows from the beginning to the end of the queue, where the first vehicle transmits an ensemble of introductions to the subsequent vehicles, such that each vehicle receives the instructions, decode, re-encode and retransmits it to the following vehicle until the message hits the end of the queue.

The use of the tensor approach in MIMO communications systems, [2]-[4], has become very popular due to the possibility to exploit more than one diversity at time, such as space, time and frequency. Another advantage of the tensor approach is that it allows us to derive either iterative or closed form semiblind receivers to jointly estimate the transmitted symbols and the transmission channel. Based on these advantages, we propose a tensor approach for Platooning systems, considering the Parallel Factor Analysis (PARAFAC) and Tucker decompositions, while using Khatri-Rao Space-Time (KRST) [7], coding and a blended strategy with Tensor Space-Time (TST) [8], and Kronecker Space-Time (KronST) [5], respectively. In order to exploit both space and time diversities, with the aim to ensure good reliability of the estimations. We propose a

Bárbara Oliveira, Universidade Federal do Ceará, Fortaleza-CE, e-mail: barbara@gtel.ufc.br; Michel Gonzaga, Universidade Federal do Ceará, FortalezaCE, email: michel.gonzaga@hotmail.com; Walter Freitas, Universidade Federal do Ceará, Fortaleza-CE, e-mail: walter@gtel.ufc.br. semiblind receiver to estimate both the transmitted symbols and transmission channel to be used for each vehicle and compare the performance of both coding strategies.

The article is organized as follows. The second section presents the theoretical fundamentals of this system, in which multilinear algebra and the platooning system are introduced. In the third section, the development of algorithms able to jointly estimate the transmitted symbols and the transmission channel are presented. The fourth section shows the Monte Carlo simulation results obtained with the proposed models. The last section discusses the conclusion of this article and some perspectives for the next steps for the study.

Notation: Scalars, vectors, matrices and tensors are denoted, respectively, by lower-case, boldface lower-case, boldface upper-case and calligraphic letters, i.e., $a, \mathbf{a}, \mathbf{A}, \mathcal{A}$. The outer product is symbolized as $\circ, \dagger$ is the Moore-Penrose pseudoinverse, $T$ represents the transposition operator, $H$ is the Hermitian transpose, the Kronecker product is represented by $\otimes$, the Khatri-Rao product is represented by $\odot$. The $n$-mode product of a tensor with a matrix is represented as $\times_{n}$.

\section{FoundaTion}

\section{A. Matrices and tensors decompositions}

In this paper, matrices and tensors decompositions are employed to model a platooning communication system. The singular value decomposition (SVD) [6], is a classic approach for matrices decompositions. Using SVD, it is possible to write a given matrix $\mathbf{A}$ in $\mathbb{C}^{I_{1} \times I_{2}}$, with $I_{1} \geq I_{2}$ as

$$
\mathbf{A}=\mathbf{U} \boldsymbol{\Sigma} \mathbf{V}^{H},
$$

where $\mathbf{U}$ is a a matrix composed by $n$ orthonormalized eigenvectors of the $n$ largest eigenvalues of $\mathbf{A}^{H} \mathbf{A}$, the matrix $\mathbf{V}$ is formed by the orthonormalized eigenvectors of $\mathbf{A} \mathbf{A}^{H}$ and $\boldsymbol{\Sigma}$ is composed by the singular values, $n$ non-negative squares roots of the eigenvalues of $\mathbf{A}^{H} \mathbf{A}, \sigma_{i}$, for $i=1, \ldots, n$. The relations of the matrices are given by

$$
\begin{array}{r}
\mathbf{U}^{H} \mathbf{U}=\mathbf{V}^{H} \mathbf{V}=\mathbf{V} \mathbf{V}^{H}=\mathbf{I}_{\mathbf{n}} \\
\boldsymbol{\Sigma}=\operatorname{diag}\left(\sigma_{1}, . ., \sigma_{n}\right) .
\end{array}
$$

A tensor [9] can be defined as an $N$-th order array $\mathcal{X} \in$ $\mathbb{C}^{I_{1} \times I_{2} \times \ldots \times I_{N}}$. An important class of tensors are the rankone tensor, defined as the outer product of $N$ column vectors $\mathbf{a}^{(n)} \in \mathbb{C}^{I_{n} \times 1}$, for $n=1,2, \ldots, N$ :

$$
\mathcal{X}=\mathbf{a}^{(1)} \circ \mathbf{a}^{(2)} \circ \ldots \circ \mathbf{a}^{(N)} .
$$

It can be defined elementwise, as well: 


$$
x_{i_{1}, i_{2} \ldots, i_{N}}=a_{i_{1}}^{(1)} a_{i_{2}}^{(2)} \ldots a_{i_{N}}^{(N)}
$$

where $1 \leq i_{n} \leq I_{N}$.

The tensors decompositions used in this work are the Parallel Factor Analysis (PARAFAC) and the Tucker decompositions. The PARAFAC decomposition model emerges of a sum of rank-one tensors. Given a rank- $R$, a third-order PARAFAC tensor $\mathcal{X} \in \mathbb{C}^{I_{1} \times I_{2} \times I_{3}}$, with elements $x_{i_{1} i_{2} i_{3}}$, it can be expressed as follows:

$$
\begin{array}{r}
\mathcal{X}=\mathbf{a}_{1} \circ \mathbf{b}_{2} \circ \mathbf{c}_{3}+\mathcal{E}, \\
\mathcal{X}=\mathcal{I}_{R} \times{ }_{1} \mathbf{A} \times{ }_{2} \mathbf{B} \times{ }_{3} \mathbf{C}+\mathcal{E}, \\
x_{i_{1}, i_{2}, i_{3}}=\sum_{r=1}^{R} a_{i_{1}, r} b_{i_{2}, r} c_{i_{3}, r}+e_{i_{1}, i_{2}, i_{3}},
\end{array}
$$

where, $\mathbf{A} \in \mathbb{C}^{I_{1} \times R}$ with elements $a_{i_{1}, r}, \mathbf{B} \in \mathbb{C}^{I_{2} \times R}$, with elements $b_{i_{2}, r}$ and $\mathbf{C} \in \mathbb{C}^{I_{3} \times R}$, with elements $c_{i_{3}, r}$, which are called the factor matrices and $\mathcal{I}_{R}$ is an identity tensor whose dimensions are $(R \times R \times R)$. The dimension $R$ stands for the number of components used in the model and $e_{i_{1}, i_{2}, i_{3}}$, elements of the tensor $\mathcal{E} \in \mathbb{C}^{I_{1} \times I_{2} \times I_{3}}$, is the residual part.

The tensor may be defined as well by its flat $n$-mode matrix unfoldings $\mathbf{X}_{I_{1} \times I_{3} I_{2}}, \mathbf{X}_{I_{2} \times I_{3} I_{1}}$ and $\mathbf{X}_{I_{3} \times I_{2} I_{1}}$, as

$$
\begin{aligned}
& \mathbf{X}_{I_{1} \times I_{3} I_{2}} \approx \mathbf{A}(\mathbf{C} \odot \mathbf{B})^{T} ; \\
& \mathbf{X}_{I_{2} \times I_{3} I_{1}} \approx \mathbf{B}(\mathbf{C} \odot \mathbf{A})^{T} ; \\
& \mathbf{X}_{I_{3} \times I_{2} I_{1}} \approx \mathbf{C}(\mathbf{B} \odot \mathbf{A})^{T} .
\end{aligned}
$$

The Tucker model [9] decomposes a tensor into loading matrices and a core tensor. Each matrix has factors in different modes. A Tucker decomposition for a third-order tensor is given by:

$$
\begin{aligned}
\mathcal{X} & =\mathcal{G} \times{ }_{1} \mathbf{A} \times{ }_{2} \mathbf{B} \times{ }_{3} \mathbf{C}+\mathcal{E} \\
\mathbf{x}_{i_{1}, i_{2}, i_{3}} & =\sum_{p=1}^{P} \sum_{q=1}^{Q} \sum_{r=1}^{R} \mathbf{a}_{i_{1}, p} \mathbf{b}_{i_{2}, q} \mathbf{c}_{i_{3}, r} \mathbf{g}_{p, q, r}+\mathbf{e}_{i_{1}, i_{2}, i_{3}} .
\end{aligned}
$$

Here $\mathbf{a}_{i_{1}, p}, \mathbf{b}_{i_{2}, q}$ and $\mathbf{c}_{i_{3}, r}$ are the elements of the matrices $\mathbf{A} \in \mathbb{C}^{I_{1} \times P}, \mathbf{B} \in \mathbb{C}^{I_{2} \times Q}$ and $\mathbf{C} \in \mathbb{C}^{I_{3} \times R}$. Also, $\mathbf{g}_{p, q, r}$ is an element of the core tensor $\mathcal{G} \in \mathbf{C}^{P \times Q \times R}$ and $\mathcal{E}$ represents the residual part of the decomposition.

Its flat $n$-mode matrix unfoldings are $\mathbf{X}_{I_{1} \times I_{3} I_{2}}, \mathbf{X}_{I_{2} \times I_{3} I_{1}}$ and $\mathbf{X}_{(3)}$ :

$$
\begin{aligned}
& \mathbf{X}_{I_{1} \times I_{3} I_{2}} \approx \mathbf{A G}_{P \times R Q}(\mathbf{C} \otimes \mathbf{B})^{T} \\
& \mathbf{X}_{I_{2} \times I_{3} I_{1}} \approx \mathbf{B G}_{Q \times R P}(\mathbf{C} \otimes \mathbf{A})^{T} \\
& \mathbf{X}_{I_{3} \times I_{2} I_{1}} \approx \mathbf{C G}_{R \times Q P}(\mathbf{B} \otimes \mathbf{A})^{T}
\end{aligned}
$$

\section{B. The Platooning system}

The Platooning system [10] allows that groups of vehicles be able to move together in an automatized way. There are vehicles called Platooning Members (PM), which receive and retransmit information, transmitted periodically to maintain the platoon safety and stability from a vehicle called the Platooning Leader (PL). The information is referred to as Cooperative Awareness Messages (CAMs) [11], including data on environmental conditions, such as desired acceleration, required distance and status of communication between the vehicles.

There are two schemes for this model, the sequential and the simultaneous transmission. In the first type, the data are transmitted in sequence, the PL transmits to the first PM and then each $\mathrm{PM}_{k}$ transmits to the following $\mathrm{PM}_{k+1}$, for $k=1, \ldots, K-1$. In the simultaneous transmission, since the acceleration data in the CAM is computed in the previous period, it is considered that an order is not necessary for the transmission, so all the PMs receive the CAM.

The general system is composed of two big steps [1]. The former is the communication between PL to PM. The PL sends the information once to the PM directly behind it and the designated receiver decodes the information, which is assigned to it. Each of the platoon members has an ID address at the application layer, so the information is sent to the desired vehicle. The second step is PM to PM communication. In this step, the PM sends the CAM to the next PM in the order defined by the platooning. After the last PM has received the data, they can compute the accurate acceleration, using the actual acceleration and the measured distance, for the next period.

\section{SySTEM MODEL}

In this paper, we propose two tensor-based models for platooning systems: one based on PARAFAC and other based on Tucker tensor decomposition. The first one allows the use of a KRST coding strategy [5] and [7], while the other one combines the TST and KronST coding strategies. These coding strategies were chosen considering the structure of the matrix unfoldings of each tensor decomposition, such that it allows us to effectively model the received signal employing these decompositions. It is considered that each member has $M$ antennas to perform both transmission and reception and the data send by the PL is received only by the first PM $\left(\mathrm{PM}_{1}\right)$. The sequential CAM transmission scheme and the cooperative protocol decode-and-forward (DF) are assumed.

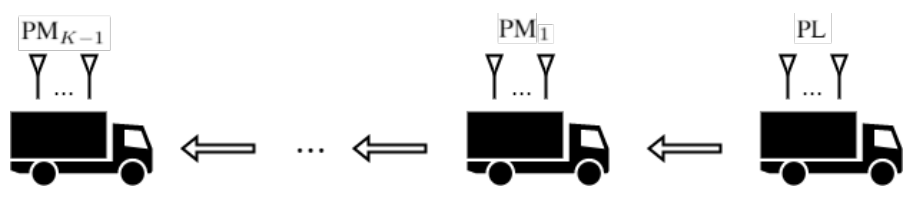

Fig. 1: Platooning System.

The general system model is represented in Fig. 1. The scenario is composed of $K$ vehicles, each of them having, respectively, $M$ antennas. A different channel $\mathbf{H}^{(k)} \in \mathbb{C}^{M \times M}$ is attributed to each pair of vehicles.

In the DF protocol [12], the PM decode and then reencode to forward the message received. The signal received by $k$-th PMs is given by:

$$
\mathbf{Y}^{(k)}=\mathbf{H}^{(k)} \mathbf{X}^{(k-1) T}+\mathbf{N}^{(k)}
$$

where $\mathbf{X}^{(k-1)}$ is the encoded transmitted signal and $\mathbf{Y}^{(k)}$ the signal received at each $\mathrm{PM}, \mathbf{H}^{(k)}$ is the $k$-th hop channel, between the $(k-1)$-th PM and the $k$-th PM. 


\section{A. Khatri-Rao Space-Time (KRST) coding}

1) Enconding: At a first approach, we consider a symbol matrix defined as $\mathbf{S} \in \mathbb{C}^{N \times M}$, with $N$ data streams with $M$ symbols each, to represent the CAM. The symbol matrix is encoded using the KRST encoding strategy [7] at each vehicle, by means of the Khatri-Rao product between a space-time code matrix $\mathbf{C}^{(k)} \in \mathbb{C}^{P \times M}$ and $\mathbf{S}$, where we assume that each $k$-th vehicle uses its code matrix. $\mathbf{C}^{(k)}$ is assumed to be a column orthonormal matrix and it follows that

$$
\mathbf{C}^{(k) \dagger}=\left(\mathbf{C}^{(k) H} \mathbf{C}^{(k)}\right)^{-1} \mathbf{C}^{(k) H}=\mathbf{C}^{(k) H} .
$$

The dimension $P$ is the row-wise spreading length, where each symbol is repeated $P$ times. The encoded symbol matrix in the PL is written as:

$$
\mathbf{X}_{M \times P N}^{(0)}=\left(\mathbf{C}^{(0)} \odot \mathbf{S}\right) .
$$

The signal received at the first PM, which was transmitted through the channel $\mathbf{H}^{(1)}$, is represented by

$$
\mathbf{Y}_{M \times P N}^{(1)}=\mathbf{H}^{(1)}\left(\mathbf{C}^{(0)} \odot \mathbf{S}\right)^{T}+\mathbf{N}_{M \times P N}^{(1)} .
$$

where $\mathbf{N}_{M \times P N}^{(1)}$ stands for the noise contribution.

The matrix $\mathbf{Y}^{(1)_{M \times P N}}$ is the flat 1-mode matrix unfolding of the tensor $\mathcal{Y}^{(1)} \in \mathbb{C}^{M \times N \times P}$, which satisfies a PARAFAC decomposition, according to the equation (9), whose mode- $n$ representation is

$$
\mathcal{Y}^{(1)}=\mathcal{I}_{M} \times{ }_{1} \mathbf{H}^{(1)} \times{ }_{2} \mathbf{S} \times{ }_{3} \mathbf{C}^{(0)}+\mathcal{N}
$$

Since the transmission rate stands for the ratio of the number of transmitted symbols, $M(N-1)$, to the number of symbol periods, $P N$, times $\log _{2} \mu$, where $\mu$ is the cardinality of the modulation, for this model it is given by the following equation

$$
T_{r}=\frac{M(N-1)}{P N} \log _{2} \mu .
$$

The same reasoning can be applied, without loss of generality, to the following $\mathrm{PM}_{k}$.

2) Decoding: The signal received at $\mathrm{PM}_{k}$, according to the DF protocol, is described as

$$
\mathbf{Y}_{M \times P N}^{(k)}=\mathbf{H}^{(k)}\left(\mathbf{C}^{(k-1)} \odot \hat{\mathbf{S}}\right)^{T}+\mathbf{N}_{M \times P N}^{(k)},
$$

where $\hat{\mathbf{S}}$ is the symbol matrix estimated in the $(k-1)$-th $\mathrm{PM}$ and sent to the $k$-th PM.

Consider its flat 3-mode matrix unfolding

$$
\mathbf{Y}_{P \times N M}^{(k)}=\mathbf{C}^{(k-1)}\left(\hat{\mathbf{S}} \odot \mathbf{H}^{(k)}\right)^{T}+\mathbf{N}_{P \times N M}^{(k)} .
$$

The factor $\left(\hat{\mathbf{S}} \odot \mathbf{H}^{(k)}\right)^{T}$ can be estimated performing a least squares (LS) estimation using the column orthonormal property of the coding matrix $\mathbf{C}^{(k)}$

$$
\mathbf{C}^{(k) H} \mathbf{Y}_{P_{k} \times N M}^{(k)}=\left(\hat{\mathbf{S}} \odot \mathbf{H}^{(k)}\right)^{T} .
$$

Since we perform a left inverse, it implies that $\mathbf{C}^{(k-1)}$ must have full column rank, such that: $M \leq P$.

Then, the matrices $\hat{\mathbf{S}}$ and $\mathbf{H}^{(k)}$ are estimated using KhatriRao factorization SVD algorithm [5].
3) Uniqueness and scale ambiguity elimination: In this decomposition, the condition of uniqueness [2] is given by

$$
\sum_{n=1}^{N} k_{\mathbf{A}^{(n)}} \leq 2 R+(N-1),
$$

in which $k_{A(n)}$ is the $k$-rank, the maximum value for $k$ columns be linearly independent, of the $N$-way tensor $\mathcal{X}$, with $\mathbf{A}^{(n)}$ components matrices and rank $\mathrm{R}$.

If the condition (26) is satisfied, the matrix factors $\mathbf{A}^{(n)}$, which for this model are the matrices $\mathbf{H}^{(k)}, \mathbf{C}^{(k)}$ and $\mathbf{S}$, for $k=1, \ldots, K-1$, are unique up to permutation and scaling ambiguity, when the factors of the tensor can be mixed and the vectors in rank-one representation can be scaled [4],

$$
\hat{\mathbf{A}}^{(n)}=\mathbf{A}^{(n)} \boldsymbol{\Pi} \boldsymbol{\Lambda}^{(n)} .
$$

In which $\Pi \in \mathbb{C}^{R \times R}$ is the permutation matrix and $\boldsymbol{\Lambda}^{(n)} \in$ $\mathbb{C}^{R \times R}$ are diagonal scaling matrices, where $\sum_{n=1}^{N} \boldsymbol{\Lambda}^{(n)}=\mathbf{I}$.

Since we assume the knowledge of $\mathbf{C}^{(k)}$, we only have the scaling ambiguities affecting our estimated matrices. Then, the final estimates are given using a scaling ambiguity factor $\omega$,

$$
\left[\mathbf{S}_{:, 1}, \mathbf{S}_{:, 2}, \ldots, \mathbf{S}_{:, M}\right] \leftarrow\left[\omega_{1} \mathbf{S}_{:, 1}, \omega_{2} \mathbf{S}_{:, 2}, \ldots, \omega_{M} \mathbf{S}_{:, M}\right],
$$

$\left[\hat{\mathbf{H}}_{:, 1}, \hat{\mathbf{H}}_{:, 2}, \ldots, \hat{\mathbf{H}}_{:, M}\right] \leftarrow\left[\omega_{1}^{-1} \hat{\mathbf{H}}_{:, 1}, \omega_{2}^{-1} \hat{\mathbf{H}}_{:, 2}, \ldots, \omega_{M}^{-1} \hat{\mathbf{H}}_{:, M}\right]$

\section{B. Kronecker Space-Time (KronST) + Tensor Space-Time (TST) coding}

1) Encoding: In this model, it is introduced a mixed coding strategy to the system, using a TST coding simultaneously with the KronST coding strategy. We consider again a symbol matrix $\mathbf{S} \in \mathbb{C}^{N \times M}$, with $N$ data streams and $M$ periods of symbols, which is encoded, at first, using the KronST by means of the Kronecker product between a Space-Time code matrix $\mathbf{C}^{(k)} \in \mathbb{C}^{P \times Q}$ and $\mathbf{S}$. In the sequence, we perform the TST coding, by taking the flat 1 -mode matrix unfolding of a Space-Time tensor $\mathcal{G}^{(k)} \in \mathbb{C}^{M \times M \times Q}, \mathbf{G}_{M \times Q M}^{(k)}$ and multiplying it with the transpose of the result of the KronST coding. We assume that each $k$-th vehicle uses its own $\mathbf{C}^{(k)}$ and $\mathcal{G}^{(k)}$. Here, it is assumed that both $\mathbf{C}^{(k)}$ and $\mathbf{G}_{O \times M^{2}}^{(k)}$ are column orthonormal matrices. The dimension $P$ and $Q$ are the row-wise and column-wise spreading lengths, respectively, where each symbol is repeated $P Q$ times. The encoded symbol matrix in the PL is written as:

$$
\mathbf{X}_{M \times P N}^{(0)}=\mathbf{G}_{M \times Q M}^{(0)}\left(\mathbf{C}^{(0)} \otimes \mathbf{S}\right)^{T} .
$$

The signal received at the first PM, which was transmitted through the channel $\mathbf{H}^{(1)}$ is given by

$$
\mathbf{Y}_{M \times P N}^{(1)}=\mathbf{H}^{(1)} \mathbf{G}_{M \times Q M}^{(0)}\left(\mathbf{C}^{(0)} \otimes \mathbf{S}\right)^{T}+\mathbf{N}_{M \times P N}^{(1)},
$$

where $\mathbf{N}_{M \times P N}^{(1)}$ stands for the noise contribution. The equation (31) can be seen as a flat 1-mode matrix unfolding of a tensor $\mathcal{Y}^{(1)}$, that satisfies a Tucker 3 decomposition, whose mode- $n$ representation is 


$$
\mathcal{Y}^{(1)}=\mathcal{G}^{(0)} \times{ }_{1} \mathbf{H}^{(1)} \times{ }_{2} \hat{\mathbf{S}} \times{ }_{3} \mathbf{C}^{(0)}+\mathcal{N}^{(1)}
$$

Since number of transmitted symbols is $M N-1$ and the number of symbol periods is $P N$, for this model the transmission rate is given by the following equation

$$
T_{r}=\frac{N M-1}{P N} \log _{2} \mu .
$$

The same reasoning can be applied, without loss of generality, to any $\mathrm{PM}_{k}$.

2) Decoding: Taking the third unfolding, with $\mathbf{C} \in$ $\mathbb{C}^{R \times P M s}$ defined as:

$$
\mathbf{Y}_{P \times N M}^{(k)}=\mathbf{C}^{(k-1)} \mathbf{G}_{Q \times M^{2}}^{(k-1)}\left(\hat{\mathbf{S}} \otimes \mathbf{H}^{(k)}\right)^{T}+\mathbf{N}_{P \times N M} .
$$

The Kronecker product $(\hat{\mathbf{S}} \otimes \mathbf{H})^{T}$ can be estimated using the least squares (LS) algorithm, we have the solution as

$$
\left(\mathbf{G}^{(k-1)} \mathbf{C}^{(k-1)}\right)^{\dagger} \mathbf{Y}_{P \times N M}=(\hat{\mathbf{S}} \otimes \mathbf{H})^{T} .
$$

where $\left(\mathbf{G}^{(k-1)} \mathbf{C}^{(k-1)}\right)$ must have full column rank, which implies that $M^{2} \leq P$.

Then, similarly to the model with KRST code, we use the Kronecker factorization SVD algorithm, [5], to obtain estimated versions of $\mathbf{S}$ and $\mathbf{H}$.

3) Uniqueness and scale ambiguity: : The uniqueness in Tucker model [4] is guaranteed when the core tensor $\mathcal{G}$ is known. If this condition is achieved, the factors are unique up to permutation and scaling ambiguity in

$$
\hat{\mathbf{A}}^{(n)}=\Psi_{i}^{(n)} \mathbf{A}^{(n)} .
$$

Where $\Psi_{i}^{(n)}$ is the scalar ambiguity for the i-th mode of $\mathcal{A}^{(n)}$ and $\sum_{n=1}^{N} \Psi_{i}^{(n)}=1$.

The, the final estimates are given using scaling ambiguity factor $\omega$,

$$
\begin{array}{r}
\omega=\hat{\mathbf{S}}(1,1) / \mathbf{S}(1,1) ; \\
\hat{\mathbf{S}} \leftarrow \hat{\hat{\mathbf{S}}} / \omega ; \\
\hat{\mathbf{H}} \leftarrow \hat{\mathbf{H}} \omega .
\end{array}
$$

\footnotetext{
Algorithm 1: Estimation of $\mathrm{H}$ and $\mathrm{S}$ in each platoon member

1 Using the received $\hat{\mathcal{Y}}$ in the PM and the known $\mathbf{C}$

2. Calculate the factor $\left(\mathbf{S} \odot \mathbf{H}_{\mathbf{k}}\right)$ using LS, solving

(24) or $(\mathbf{S} \otimes \mathbf{H})$, solving (32)

3. Compute the Khatri-Rao Factorization SVD or the

Kronecker Factorization SVD

4. Eliminate the scaling ambiguities using (27) and (28) or using (34), (35) and (36).

5 . Estimate the received symbols.

6. Use the DF protocol, with the actual member coding the $\hat{\mathbf{S}}$ with the defined code matrix $\mathbf{C}$ and transmitting to the next PM.
}
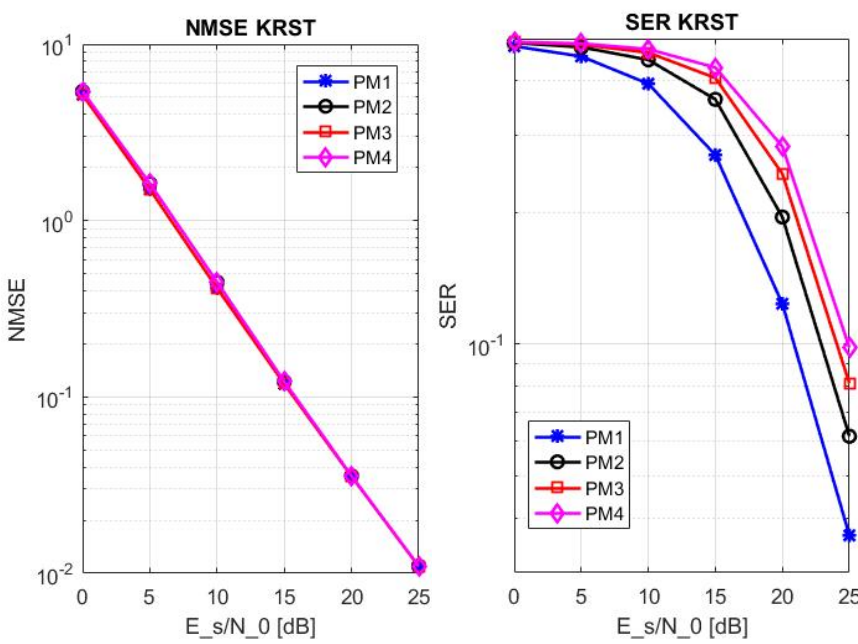

(a) Channel NMSE KRST (b) SER KRST Platooning Platooning comparison comparison

Fig. 2: Simulation Results KRST

\section{REsults}

This section aims to provide some computational simulations, in order to evaluate the performance of both models and their respective estimation algorithms. This section contains the Monte Carlo simulation method for the estimation of the transmission symbols, over a range of signal-to-noise ratio (SNR) values.

In the scenario, we have used a 64-QAM modulation. It was assumed the Rayleigh fading channel and the white Gaussian additive noise with variance $\sigma^{2}$, the matrices $\mathbf{C}^{(k)}$ and $\mathbf{G}_{Q \times M^{2}}^{(k)}$ are assumed to be truncated DFT matrices. Then, to compare the performance of the simulations, the symbol error rate (SER) was calculated for each PM and plotted against the symbol energy to noise spectral density ratio $\left(E_{s} / N_{0}\right)$ values. It was also considered the normalized mean square error (NMSE) of the estimated channels of the models presented in this paper, also as a function of the $E_{s} / N_{0}$,

$$
\operatorname{NMSE}(\mathbf{H})=\frac{1}{M} \sum_{m=1}^{M} \frac{\left\|\hat{\mathbf{H}}_{\mathbf{m}}-\mathbf{H}_{m}\right\|_{F}^{2}}{\left\|\mathbf{H}_{m}\right\|_{F}^{2}}
$$

where $\hat{\mathbf{H}}_{m}$ is the estimated version of $\mathbf{H}_{m}$ at each $m$-th run.

The parameters of the simulations were chosen in order to ensure the same transmission rate for both strategies. For the KRST we have $\mathrm{N}=2, \mathrm{M}=3, \mathrm{P}=4$ and for the KronST + TST we have $\mathrm{N}=2, \mathrm{M}=2, \mathrm{P}=4, \mathrm{Q}=4$.

\section{A. Symbols and channel performances for different PMs}

Here the impact of the retransmission of the transmitted symbols is evaluated over four PMs, considering the KRST in the Figures 2(a) and 2(b) and the combination of the KronST and TST coding strategies in the figures 3(a) and 3(b).

As it can be seen in the Figures 2(b), 3(b), as one may expect, for each coding strategy the SER is degraded once the symbols are retransmitted over the PMs, since what is 

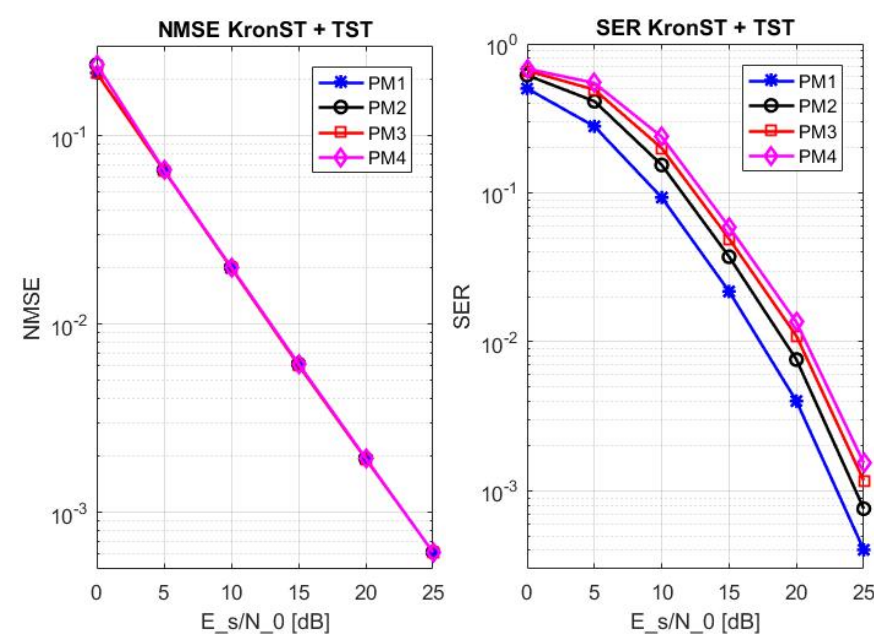

(a) Channel NMSE KronST + (b) SER KronST + TST PlaTST comparison KronST tooning comparison

Fig. 3: Simulation Results KronST + TST

transmitted is an estimated version of the transmitted symbols recovered at each PM and encoded again and transmitted to the next PM. One may notice, that according to the figure 2(a), 3(a) the estimation of the transmission channel, for each coding strategy, presents the same performance for each PM, which is also expected since the channel between the PMs have the same characteristics.

\section{B. Coding strategies comparison}

Here the performance of the symbols and channel estimation for the two proposed coding strategies are compared. Figure 4(a) shows the SER comparison for each strategy, one may notice that the combined use of the TST and KronST shows the best performance, especially for high values of $E_{s} / N_{0}$. The figure 4(b) compares the channel NMSE for each strategy and its results collaborate with the hypothesis that the second coding strategy presents the best performances, since it is also the case for the channel estimation.

\section{CONCLUSIONS}

In this work, we investigate the application of KRST and combined TST and KronST coding techniques for vehicle platooning systems. Through the comparative analysis between the models, we see that the second achieved the best performance. This can be explained by the increased redundancy that the model offers. The observed results also contribute to confirm the effectiveness of using multidimensional algebra tools in scenarios of mobile communications.

Further work will be done and better results might be achieved with different configurations, using more sophisticated tensor decompositions and different techniques of signal transmission, like Orthogonal Frequency-Division Multiplexing (OFDM).
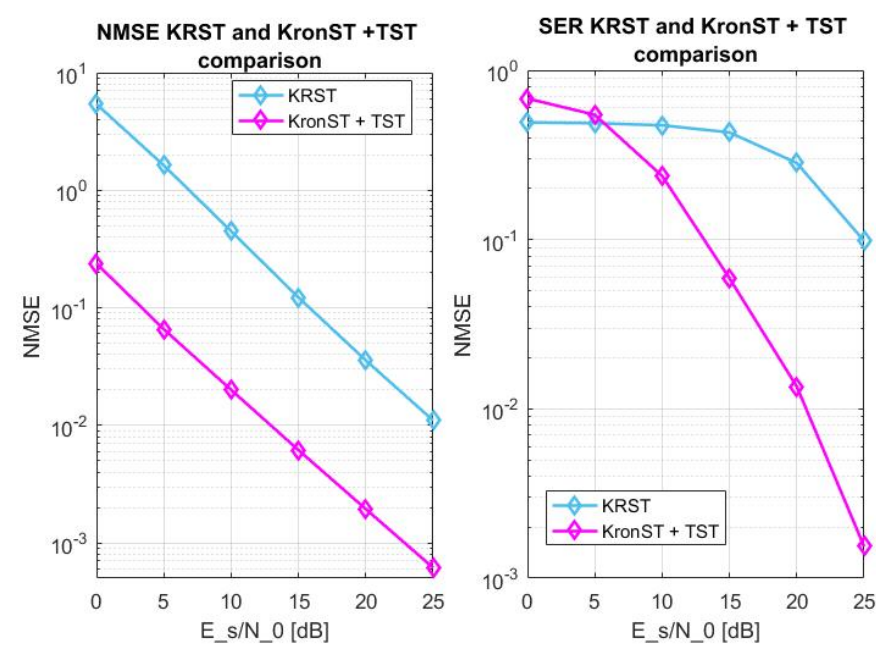

(a) SER comparison between (b) SER comparison between the KronST+TST and KRST the KronST+TST and KRST

Fig. 4: Simulation Results KronST+TST Vs. KRST

\section{ACKNOWLEDGEMENTS}

The authors are thankful to Coordenação de Aperfeiçoamento de Pessoal de Nível Superior CAPES - Brasil for partially supporting this work (Finance Code 001). The research of Walter da Cruz Freitas Jr. is partially supported by CNPq.

\section{REFERENCES}

[1] G. Nardini, A. Virdis, C. Campolo, A. Molinaro, G. Stea. Cellular-V2X Communications for Platooning: Design and Evaluation. Sensors, 2018.

[2] N. D. Sidiropoulos, G. B. Giannakis, and R. Bro, "Blind PARAFAC receivers for DS-CDMA systems," IEEE Transactions on Signal Processing, vol. 48, no. 3, pp. 810-823, Mar 2000.

[3] X. Hang , A.L.F. de Almeida, Z. Yang. Channel estimation for MIMO multi-relays systems using a tensor approach. EURASIP Journal on Advances in Signal Processing, 2014.

[4] D. Rocha. Nested tensor decomposition applied to cooperative MIMO communication systems. Thesis (doctoral) - Universidade Federal do Ceará, 2019

[5] W. C. Freitas Jr., G. Favier, A. L. F. de Almeida. Generalized KhatriRao and Kronecker Space-Time Coding for MIMO Relays Systems with Closed-Form Semi-Blind Receivers. Signal Processing, 2018.

[6] G. H. Golub, C. Reinsch .Singular Value Decomposition and Least Squares Solutions. In: F. L. Bauer, A. S. Householder, F. W. J. Olver, H. Rutishauser , K. Samelson , E. Stiefel. Handbook for Automatic Computation. Die Grundlehren der mathematischen Wissenschaften (in Einzeldarstellungen mit besonderer Berücksichtigung der Anwendungsgebiete), vol 186. Springer, Berlin, Heidelberg, 1971.

[7] N. D. Sidiropoulos, R. S. Budampati , Khatri-Rao space-time codes, IEEE Trans. Signal Process, 2002.

[8] G. Favier , M. N. da Costa , A. L .F. de Almeida , J. M. T. Romano, Tensor space-time (TST) coding for MIMO wireless communication systems, Signal Processing, v.92 n.4, p.1079-1092, April, 2012

[9] T. G. Kolda and B. W. Bader, "Tensor decompositions and applications," SIAM review, 2009.

[10] V. Vukadinovic, K. Bakowski, P. Marsch, I. Garcia, H. Xu, M. Sybis, P. Sroka, K. Wesolowski, DavidLister, I. Thibault. 3GPP C-V2X and IEEE 802.11p for Vehicle-to-Vehicle communications in highway platooning scenarios. Ad Hoc Networks, 2018.

[11] V. de Cózar, J. Poncela , M. Aguilera , M. Aamir, B.S. Chowdhry Cooperative Vehicle-to-Vehicle Awareness Messages Implementation. Communications in Computer and Information Science, 2013.

[12] G. Levin, S. Loyka. Amplify-and-Forward Versus Decode-and-Forward PMing: Which is Better?.

[13] N. D. Sidiropoulos and R. S. Budampati. Khatri-Rao space-time codes. IEEE Transactions on Signal Processing, 2002. 